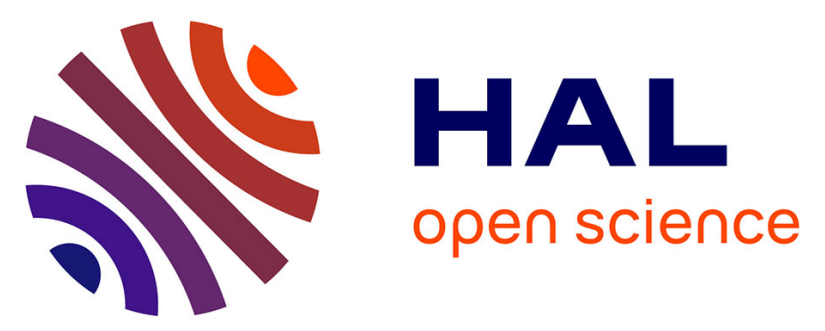

\title{
Tumor cell load and heterogeneity estimation from diffusion-weighted MRI calibrated with histological data: an example from lung cancer
}

\author{
Yi Yin, Oliver Sedlaczek, Benedikt Müller, Arne Warth, Margarita \\ González-Vallinas, Bernd Lahrmann, Niels Grabe, Hans-Ulrich Kauczor, Kai \\ Breuhahn, Irene Vignon-Clementel, et al.
}

\section{To cite this version:}

Yi Yin, Oliver Sedlaczek, Benedikt Müller, Arne Warth, Margarita González-Vallinas, et al.. Tumor cell load and heterogeneity estimation from diffusion-weighted MRI calibrated with histological data: an example from lung cancer. IEEE Transactions on Medical Imaging, 2017, 10.1109/TMI.2017.2698525 . hal-01421398

\section{HAL Id: hal-01421398 \\ https://hal.inria.fr/hal-01421398}

Submitted on 2 May 2017

HAL is a multi-disciplinary open access archive for the deposit and dissemination of scientific research documents, whether they are published or not. The documents may come from teaching and research institutions in France or abroad, or from public or private research centers.
L'archive ouverte pluridisciplinaire HAL, est destinée au dépôt et à la diffusion de documents scientifiques de niveau recherche, publiés ou non, émanant des établissements d'enseignement et de recherche français ou étrangers, des laboratoires publics ou privés.

$$
\text { Copyright }
$$




\title{
Tumor cell load and heterogeneity estimation from diffusion-weighted MRI calibrated with histological data: an example from lung cancer
}

\author{
Yi Yin, Oliver Sedlaczek, Benedikt Müller, Arne Warth, Margarita González-Vallinas, Bernd \\ Lahrmann, Niels Grabe, Hans-Ulrich Kauczor, Kai Breuhahn, Irene E. Vignon-Clementel, Dirk Drasdo*
}

\begin{abstract}
Diffusion-weighted magnetic resonance imaging (DWI) is a key non-invasive imaging technique for cancer diagnosis and tumor treatment assessment, reflecting Brownian movement of water molecules in tissues. Since densely packed cells restrict molecule mobility, tumor tissues produce usually higher signal (a.k.a less attenuated signal) on isotropic maps compared to normal tissues. However, no general quantitative relation between DWI data and the cell density has been established.

In order to link low-resolution clinical cross-sectional data with high-resolution histological information, we developed an image processing and analysis chain, which was used to study the correlation between the diffusion coefficient ( $D$ value) estimated from DWI and tumor cellularity from serial histological slides of a resected non-small cell lung cancer (NSCLC) tumor. Color deconvolution followed by cell nuclei segmentation was performed on digitized histological images to determine local and cell-type specific 2d (two-dimensional) densities. From these the 3d
\end{abstract}

Manuscript received July 15, 2016. This research was supported by the BMBF-projects CancerSys/LungSys, which is funded by the German Federal Ministry of Education and Research under Grant FKZ 0316042H and 0316042B. YY further acknowledges support from EU-NOTOX, DD from ANR-INVADE, and MGV from the Alfonso Martin Escudero Foundation. Asterisk indicates corresponding author.

This paper has supplementary downloadable material available at http://ieeexplore.ieee.org, provided by the authors.

Y. Yin, I. E. Vignon-Clementel and *D. Drasdo are at INRIA Paris, and Sorbonne Universités UPMC Univ. Paris 6, Laboratoire Jacques-Louis Lions, Paris, France (e-mail: yi.yin@inria.fr; Irene.Vignon-Clementel@inria.fr; dirk.drasdo@inria.fr).

O. Sedlaczek and HU Kauczor are at the Translational Lung Research Center Heidelberg (TLRC), member of the German Centre for Lung Research (DZL), Department of Diagnostic \& Interventional Radiology, University Hospital of Heidelberg, and Department of Diagnostic and Interventional Radiology with Nuclear Medicine, Thoraxklinik at University of Heidelberg, and the DKFZ, Department of Diagnostic Radiology, Heidelberg, Germany (e-mail: o.sedlaczek@dkfz-heidelberg.de; hu.kauczor@med.uni-heidelberg.de).

B. Müller, M. González-Vallinas, A. Warth, and K. Breuhahn are from the Institute of Pathology, University Hospital Heidelberg, Germany (e-mail: benedikt.f.mueller@gmx.de; Margarita.Gonzalez@med.uni-heidelberg.de Arne.Warth@med.uni-heidelberg.de; Kai.Breuhahn@med.uni-heidelberg.de).

A. Warth is also at the Translational Lung Research Center Heidelberg (TLRC), member of the German Centre for Lung Research (DZL).

B. Lahrmann and N. Grabe are at the Institute of Pathology, University Hospital Heidelberg, and Department of Medical Oncology, NCT, University Hospital Heidelberg, Germany (e-mail: Bernd.Lahrmann@bioquant.uni-heidelberg.de;

Niels.Grabe@bioquant.uni-heidelberg.de).

*D. Drasdo is also at IZBI, University of Leipzig, Leipzig, Germany DD.

Shared first authorship: YY and OS; Shared last authorship: KB, IEVC, and

Copyright (c) 2017 IEEE. Personal use of this material is permitted. Permission from IEEE must be obtained for all other users, including reprinting/ republishing this material for advertising or promotional purposes, creating new collective works for resale or redistribution to servers or lists, or reuse of any copyrighted components of this work in other works. (three-dimensional) cell density was inferred by a model-based sampling technique, which is necessary for the calculation of local and global 3d tumor cell count. Next, DWI sequence information was overlaid with high-resolution $\mathrm{CT}$ data and the resected histology using prominent anatomical hallmarks for co-registration of histology tissue blocks and non-invasive imaging modalities' data. The integration of cell numbers information and DWI data derived from different tumor areas revealed a clear negative correlation between cell density and $D$ value. Importantly, spatial tumor cell density can be calculated based on DWI data.

In summary, our results demonstrate that tumor cell count and heterogeneity can be predicted from DWI data, which may open new opportunities for personalized diagnosis and therapy optimization.

Index Terms-DWI, heterogeneity, histology data, histopathology, image analysis, tumor cellularity.

\section{INTRODUCTION}

CANCER is one of the leading causes of mortality worldwide. In the medical treatment of cancer, the histopathology of tumor specimens provides the basis for diagnosis and therapy-planning. However, biopsies reflect only very restricted tumor areas, which might be of impaired significance with regard to the majority of systemic tumor load and respective therapeutic approaches [1]. The second pillar of tumor characterization is non-invasive imaging, which yields a relatively low-resolution picture of the local tissue, mapping information from different components (such as water content, radiation attenuation, cell density, extracellular matrix density etc.) on voxel intensity values [2]. This reduction of information impedes interpretation of the images. An example for non-invasive imaging of ultrastructural information available in almost every clinical magnetic resonance imaging (MRI) is diffusion-weighted imaging (DWI). The potential of DWI on non-invasive prediction of cell density has become a research focus. DWI has been shown to be effective in assessing cancer treatment response [3]-[7]. DWI reflects the motion of water molecules in tissue. For example, higher packing of the cells is associated by a restricted random (Brownian) movement of water, which is associated by a higher (i.e., less attenuated) signal in DWI. Its voxel resolution is typically in the range of $\mathrm{mm}$ [8]. The water molecule diffusion-driven displacement levels probe the tissue structure at different scales, including intracellular, intercellular, and supra-cellular scales, the latter by sensing water molecule 
movement in vascular networks [8]. Most often cell density is higher in the solid portion of the vital part of a tumor than in normal tissue, and thus diffusion is expected to be restricted due to densely packed tumor cells [9]. However, tissue microarchitecture and composition may to some extend influence the voxel intensity value. The relation between DWI intensity and cell density is not unique for all tissues.

The intravoxel incoherent motion (IVIM) model is an advanced method to separate diffusion and perfusion in DWI, in which the signal decay in each voxel can be described by:

$$
\frac{s(b)}{s_{0}}=(1-f) \cdot \exp (-b \cdot D)+f \cdot \exp \left(-b \cdot\left(D+D^{*}\right)\right) \text {. }
$$

Here, $\mathbf{S}(b) / \mathrm{S}_{0}$ is the ratio of echo signal amplitude of diffusion-weighted to non diffusion-weighted intensity [10]. Parameter $b$ defines the degree of diffusion sensitization, depending on magnitude, duration of the gradient pulse and the time interval between two successive pulses. The precise mathematical relation depends on the shape of the pulses [11]. $\mathrm{D}$ is the diffusion coefficient, characterizing the restricted mobility of water molecules, for example in intercellular spaces $D^{*}>>D$ is the pseudo-diffusion coefficient related to blood flow in the capillary network, that in a voxel often has a pseudorandom orientation. $\mathrm{f}$ is proportional to the blood vessel volume fraction.

Different attempts have been undertaken to relate $S / S_{0}$ to tissue characteristics. A common approach uses instead of the bi-exponential function a fit to only one exponential, $S(b)$ / $S_{0}=\exp (-b \cdot A D C)$. ADC lumps together contributions from water movement by diffusion and perfusion within the voxel (and is thus called apparent diffusion coefficient) [8], [10], hence it is inherently less accurate in probing true diffusion. Changing $b$ changes the weight of the terms; at large $b$ true diffusion is probed to a greater extend.

So far, a few studies comparing DWI and histological information have been performed [12]-[17]. In [12], Kono et al. computed the average ADC vs. the expectation value of the nuclear area fraction in patients with brain tumors, representing each patients by one single point in the graph. Histological evaluation was performed on selected sample images. The same quantity was computed by Hayashida et al. [13] for metastases of different primary tumors in brain. Lyng et al. [14] considered human melanoma xenografts in mice, calculating ADC vs. manually detected cell density for different tumors and different regions within the same tumor. Anderson et al. [15] computed the ADC vs. cell volume fraction in cell suspensions and packed arrays of human glial and red blood cells. Ginat et al. [16] correlated the manual estimated cell densities with ADC values measured in different patient tumors to differentiate benign from malignant skull lesions. Schmainda [17] investigated the utility of ADC in diagnosis and treatment in glioma based on the inverse correlation between ADC and tumor and tissue cell density. In each case, a negative correlation between the ADC value and the measure characterizing cell density in the histological or in-vitro samples was found [12]-[17]. Among these studies, the histology analysis was performed either manually or by simple image processing tool that used global thresholding, which is sensitive to errors [12]. Besides, the quantitative analysis was limited to only a small number of histological slices.

Some investigators compared simulated cell density differences to relative ADC values, to estimate tumor cell numbers. The studies were based on the negative correlation and tumor growth mathematical models [18]-[21]. For example, Atuegwu et al. [18] estimated the growth rate for brain cancer in rat based upon a logistic growth model in each voxel in combination with the ADC. Weis et al. [19] estimated the cellularity in human breast cancer from a Fisher-KPP equation with the local ADC coupled to the mechanical stress modified by the growing tumor, using the maximal number of cells fitting within a voxel as fit parameter. Further applications based on [19] were proposed in [20] for response of breast cancer to neoadjuvant therapy and in [21] for glioma growth in rat brain.

Most studies address animal tumors or xenograft [14], [15], [18], [21], [22] and consider mean values for the individual tumors without statistical quantification of variation. Moreover, the ADC value lumping perfusion and diffusion was used [23]. The ADC is usually computed as the mean measurement of different regions of interest (ROIs) [12], [13], [16] or on each voxel of one large (tumor-size scale) ROI [18]-[21] on one MRI slice without further consideration of the local tumor micro-structure.

In this study we investigate both, the diffusion value D vs. $3 \mathrm{~d}$ (' $\mathrm{d}$ '=dimension) cell density relation, and the $\mathrm{D}$ vs. area fraction relation for a single patient non-small cell lung cancer (NSCLC) tumor. By using D instead of ADC we inherently address diffusion only, hence avoid lumping diffusion and perfusion together. ADC is formally equivalent to $\mathrm{D}$ when $\mathrm{f}$ is zero. In our study given the ranges of $\mathrm{b}$ and $\mathrm{f}, \mathrm{ADC}$ is not equal to $\mathrm{D}$. Besides, $\mathrm{ADC}$ is approximately equal to $\mathrm{D}$ at very large $\mathrm{b}$ values by eliminating the effects of perfusion. However, the increase of $b$ leads to a higher probability of lower SNR.

For our analysis we developed an automated image segmentation pipeline as this facilitates analysis of large histological samples necessary to reduce statistical errors. A large variety of methods have been proposed for cell nuclei segmentation on cancer histological images [24]. Popular approaches to segment cell nuclei include the Hough transform [25], voting along the direction of the image gradient to infer the center of the object [26]-[28] separating clustered nuclei along points of high concavity [29], [30], unsupervised Bayesian classification [31] and supervised methods [32]. Our algorithm combines the image local intensity minimum and gradient information to detect the cell nucleus with low staining quality, such as irregular nucleus contour, small fragments inside individual nucleus, which can be detected as multiple nuclei by the previous mentioned algorithms. We use KL1 staining as cancer cell marker to ensure identification accuracy between cancer and non-cancer cells within the tumor. The code can be downloaded at msysbio.com/cellsegmquant (integration into software TiQuant [33] is envisaged).

The automated segmentation is in-line with the aims of digital pathology. The routine analysis of tissue samples performed by pathologists is tedious, subjective, and time consuming. Tissue slides are digitized for quantitative analysis 
based on image processing techniques and automated analysis. The later increase speed and reproducibility of cancer evaluation and staging. By sampling from different locations of the same tumor we infer a relation between $D$ values vs. $3 \mathrm{~d}$ cell density and area fraction, respectively, for one single tumor alone, that span a surprisingly large range of values hence displaying a significant degree of heterogeneity. The findings suggest that tumor heterogeneity in individual patients can be detected non-invasively.

In summary, the novelties of this work comprise: study in human lung tumor with large heterogeneity; efficient algorithm for nuclei detection and application to tumor cells; proposed ellipsoid model based algorithm for $3 \mathrm{~d}$ cell density estimation; cancer and non-cancer cell-count on 350 whole histology slides; investigation of heterogeneity within an individual tumor from DWI; correlation between diffusion coefficient assessed by the IVIM model in DWI and cell density; counting of total cell number of tumor based on this correlation. The paper is structured presenting first the data acquisition, then the methods to analyze them. The results on the histological and DWI data analyses follow, along with the correlation. The paper ends with discussion of the results in light of the literature, and the limitations of the study, as well as concluding remarks.

\section{DATA ACQUiSITION}

\section{A. Patient Selection and Tissue matching}

Primary human NSCLC tissue was obtained from a patient as part of a patient cohort who underwent a lobar resection for lung cancer at the Department of Thoracic Surgery, Thoraxklinik Heidelberg-Rohrbach (Germany). Informed consent was obtained from the patient (Appendix-A). After tumor resection the patient underwent adjuvant radiation therapy.

The tumor was selected for the following reasons. It showed invasion into the pericardial fat, which made it immobile during breathing excursions, thus reducing the effects of respiratory motion during cross-sectional imaging and avoided difficulties in the identification of tumor sub-volume locations. Centrally the contact between the tumor and the large bronchial structures (diameter of about 5-6mm) could be used to reliably and easily orient the tumor after resection. The bronchial structures were located within the axial planes of the CT and MRI (Fig. 1(a), (b)), to co-register the different imaging modalities (CT and MRI).

Further serial sections were performed in parallel to the axial cut with thickness of each section of approximately $1 \mathrm{~cm}$. The tumor tissue slice (Fig. 1(c)) selected for further work-up, was the one above the central cutting line of the tumor, and contained all relevant tissue and cell types of the tumor such as normal lung tissue, vital tumor tissue, and surrounding adipose tissue. Relevant MRIs were acquired in axial plane identical to the selected tumor slice (Fig. 2), based on which the tumor tissue was classified. Identification of central necrosis was based on DCE-dynamics and $\mathrm{K}_{\text {trans }}$ (volume transfer coefficient reflecting vascular permeability) value from DCE-MRI (Fig. 2(a)) which has the same coordinate system and structural images as the other MRIs. The functionally identified borders of the necrosis (the central area being not perfused) were transferred to the high resolution T1-contrast image (Fig. 2(b)). The outer border of the tumor (Fig. 2(b)) was manually segmented based on contrast differences.

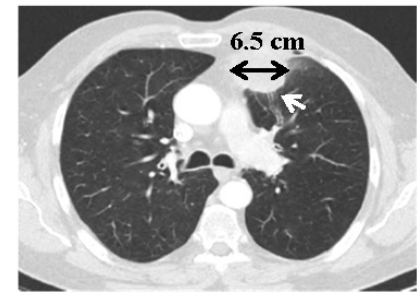

(a)

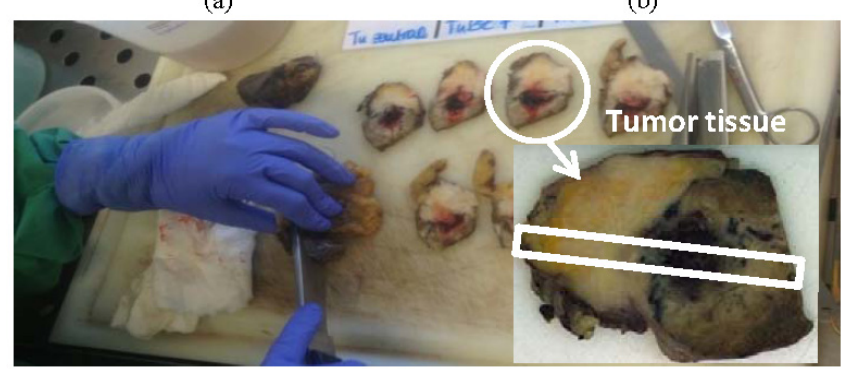

(c)

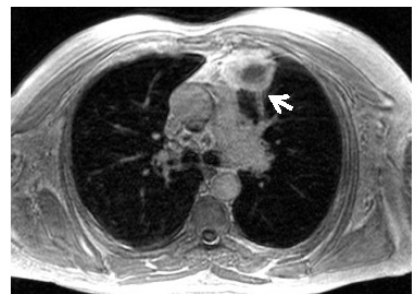

(b)
Fig. 1. Selection of patient tumor. (a) CT slice; (b) Corresponding T1-weighted MRI slice; (c) Tumor tissue slices, the one which was selected for further analysis is highlighted. The white rectangle delineates the analyzed strip (Fig.10(a)). Tumor diameter is $6.5 \mathrm{~cm}$ (black double arrow on (a)). The chronic inflammation is indicated by the enlarged diameter of the bronchial structures up to the periphery (white arrow on (b)).

For further analysis, the tumor slice was cut into smaller pieces. Seven tissue samples were cut from the tumor strip delineated by a white rectangle as shown in Fig. 1(c) and Fig. 10(a). The cutting line (Fig. 1(c)) was drawn from the extension of bronchus III through the center of the necrosis towards the most distant part of the adipose tissue invasion by the tumor. Based on the cutting line, histological samples were visually matched to fit the structures identified in MR/CT. Fine calibration was done by careful evaluation of the multimodal contrast with regard to specific tissue types in the MRI to select the pixels that correspond to the histological specimens. This procedure was several times repeated ensuring reproducibility.

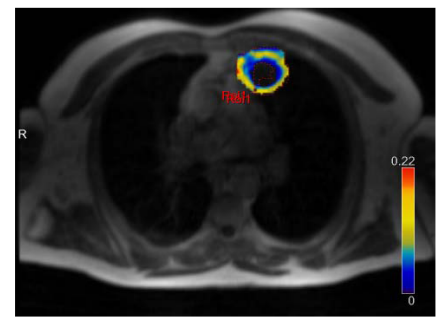

(a)

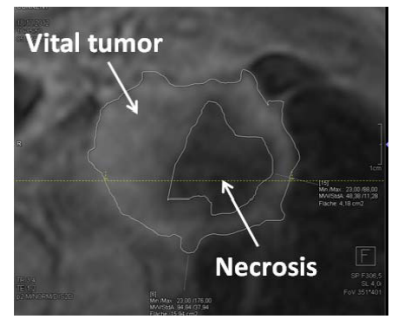

(b)
Fig. 2. Tissue classification based on functional MRI: (a) Perfusion kinetics of DCE-MRI (the colorbar indicates the $\mathrm{K}_{\text {trans }}$ values displayed in the tumor region); (b) Necrotic tumor contour (necrosis from (a)) overlaid on T1-CEFS-MRI. The outer thin line delineates the tumor region. The vital tumor is in-between. 


\section{B. DWI}

Within a clinical standard thorax MRI [34] including a tumor perfusion contrast dynamic MR-image [35] a navigated diffusion echo-planar imaging sequence was used acquiring 6 b-values above zero $=50,100,150,200,400$, and $800 \mathrm{~s} / \mathrm{mm}^{2}$. The diffusion-encoding gradients were applied in three orthogonal directions; therefore the diffusion tensor is assumed isotropic. The sequence used the following parameters: TE 75 ms, TR $8364 \mathrm{~ms}$, SL $6 \mathrm{~mm}$. Imaging was performed on a $1.5 \mathrm{~T}$ MRI (Avanto, Siemens, Erlangen). The DWI voxel resolution is $2.1 \mathrm{~mm} \times 2.1 \mathrm{~mm} \times 6 \mathrm{~mm}$. The $\mathrm{D}$ value of each voxel was obtained by fitting the voxel signal $S / S_{0}$ vs. the b-value to the above signal attenuation model (1) using the freely available software MITK Diffusion [36]. For the determination of the tissue diffusion coefficient, all above b-values were taken into account.

\section{Materials}

\section{1) Tissue sampling and processing}

After fixation of the tissue slice (about $7 \mathrm{~cm} \times 5 \mathrm{~cm} \times 0.5 \mathrm{~cm}$ ) in $4 \%$ buffered formalin overnight, the sample was cut in smaller pieces (about $1 \mathrm{~cm} \times 1 \mathrm{~cm} \times 0.5 \mathrm{~cm}$ ), transferred in paraffin, and systematically cut in $1-2 \mu \mathrm{m}$ thick sections using a microtome (HM 355, Microm, Thermo Scientific, Braunschweig, Germany). Selected samples were stained with hematoxylin/eosin (H\&E) and carefully reviewed by the study pathologist (A.W.) to determine the proportion of viable tumor cells, stromal cells, normal lung cell content, infiltrating lymphocytes, and necrotic areas.

\section{2) Sample staining}

Sections were stained using hematoxylin/eosin (H\&E) and acid fuchsin orange G (AFOG) standard protocols. In addition, an anti-pan cytokeratin antibody (clone KL1, Abcam, Cambridge, UK) was used for epitope-specific stain. In brief, staining was performed using the Dako Autostainer (Dako, Hamburg, Germany) and the following protocol: tissue slides were air-dried in an incubator at $42^{\circ} \mathrm{C}$ overnight and deparaffinized in xylene $(2 \times 10 \mathrm{~min})$. After rehydration in graded ethanol, the slides were pretreated in $0.01 \mathrm{M}$ sodium citrate $(\mathrm{pH} 6.0)$ in a pressure cooker for $10 \mathrm{~min}$. Afterwards, primary antibodies in PBS/Tween were added for $30 \mathrm{~min}$ at room temperature and slides were washed with PBS/Tween for 5 min before the secondary antibody was applied for 20 minutes (1:1.000 in PBS/Tween). The samples were then incubated with $1 \%$ hydrogen peroxide diluted in PBS/Tween ( 5 min). After signal detection using amino-ethyl-carbazol (AEC, $2 \times 7 \mathrm{~min}$ ) nuclei were stained using hematoxylin.

\section{3) Image acquisition}

Glass slides were automatically scanned in bright-field mode at a 40 -fold magnification $(0.23 \mu \mathrm{m} /$ pixel $)$ with the Hamamatsu NanoZoomer 2.0-HT Scan System (Hamamatsu Photonics, Hamamatsu, Japan). The imaging system consists of three 4096×64 pixel TDI-CCD sensors (sensor pixel size: $8 \mu \mathrm{m}^{2}$ ), providing manual/automatic adjustments of the number of focus points and multiple plane digitization [37]. For the scanning of the object slides, the slide scanner automatically detects the ROI that contains the tissue and also determines automatically a valid focal plane for scanning. Resulting virtual slides have an average compressed file size of 1 GB (JPEG, Q = 90) and uncompressed of about $25 \mathrm{~GB}$, depending on the scanned area.

\section{DATA ANAlysis Methods}

Based on the tumor DWI and histology data, we developed a processing pipeline for tumor cellularity estimation. The pipeline includes:

1. $2 \mathrm{~d}$ cell density estimation based on histological image analysis. A segmentation algorithm is proposed to detect cell nuclei in histological images. Each cell is assumed to have only one nucleus, and the $2 \mathrm{~d}$ cell density is obtained by dividing the number of detected nuclei by the area of the tissue slide. The analysis tool enables the automated quantification of cellularity in large histological samples with high accuracy.

2. $3 \mathrm{~d}$ cell density estimation in a two-step process. The first step involves the computation of $3 \mathrm{~d}$ cell densities from $2 \mathrm{~d}$ segmentations separately for cancer and non-cancer cells based on a model that approaches cell nuclei shapes by ellipsoids. Next the total $3 \mathrm{~d}$ cell density is computed involving both cancer and non-cancer cells. This is necessary to estimate the tumor cell load for the entire tumor as well as on a voxel-wise basis.

3. Correlation of the DWI-images and tumor cellularity in both $2 \mathrm{~d}$ and $3 \mathrm{~d}$ by comparison of diffusion coefficients and cell densities of seven selected tumor tissue samples. The correlation is used to calculate the tumor cell load in each voxel of the DWI data for analysis of tumor heterogeneity, which includes calculation of the cell distribution in tumor regions for which no histology data is present as well as for cell load estimation of the entire tumor.

4. Cell number estimation of the whole tumor. The tumor contour and volume are obtained by tumor segmentation in DWI data. The $3 \mathrm{~d}$ cellular distribution of the tumor can be estimated from the $\mathrm{D}$ value distribution based on the relationship between $\mathrm{D}$ value and cell density in step 3 . The total cell number is finally obtained by summing up the cell numbers in all those voxels of the DWI data that belong to the tumor.

\section{A. Histological image analysis}

We propose an automated algorithm for the detection and classification of cells in tumor tissue based on H\&E staining for all nuclei, and KL1 staining for cancer cytoplasm. The algorithm depicted in the flowchart of Fig. 3 was tested on digitized histological slides.

\section{1) Pre-processing}

The original histological image is at 40 -fold magnification with file size of larger than 1 GB. To trade-of visible details and computation time, a magnification of 20 -fold was chosen $(0.455 \mu \mathrm{m} / \mathrm{pixel})$ for all images for histology analysis. In the cutting process, the spatial correspondence between neighbor tissue slides is lost and information from different slides is difficult to compare. Before analyzing the data quantitatively, the scanned histology slides were preprocessed once with, once 
without image registration [38] (see Table I). The Gaussian blur was applied to reduce the noise in both cases. Data represented below are for registered images.

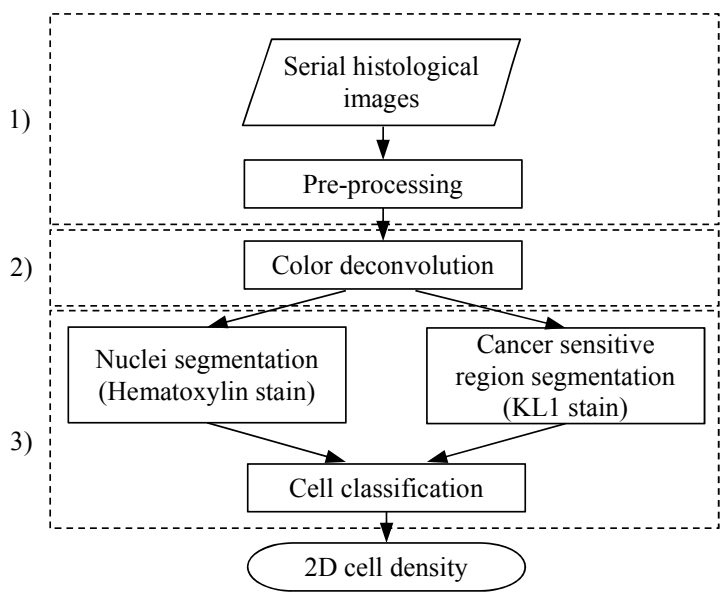

Fig. 3. Flowchart of the image analysis algorithm for automatic histology study, from serial histology slides to $2 \mathrm{~d}$ cell density evaluation. The left column indicates the corresponding sub-sections in Section III. A.

\section{2) Color deconvolution}

An automated color deconvolution [39] was performed in the histological image after pre-processing to split the color image into hematoxylin and KL1 channels. The KL1 channel image was used to generate a mask image, which separated the cancer sensitive region from the background. The final detected nuclei were then classified into cancer and non-cancer nuclei based on the mask image. From the hematoxylin channel image, we then implemented an automatic cell segmentation algorithm.

The color deconvolution method assumes that a pixel is a weighted sum of 3 stains, the two stains hematoxylin and KL1, and the background. Each stain $j$ has its own characteristic absorbance for light in each of the three RGB channels $i=\{r, g, b\}$. For channel $i$, based on Lambert-Beers law, Ruifrok et al. [40] proposed for the intensity of light after passing a tissue sample in a bright field micrograph the relation

$$
I_{i}=I_{0, i} e^{-\sum_{j} A_{i, j} C_{j}},
$$

where $I_{0, i}$ is the intensity of light entering the specimen in color channel $i=\{r, g, b\} ; A_{i, j}$ is the corresponding absorption factor, and $C_{j}$ is the concentration of the stain $j$. With incident white light $I_{0, i}=255$ for each channel $i=\{r, g, b\}$ the optical density reads

$$
\Delta_{i}=\sum_{j} A_{i, j} C_{j}=-\ln \left(\frac{I_{i}}{I_{0, i}}\right) .
$$

The optical density of the color image is expressed as $\Delta=A C$, where $\Delta$ is a three-element vector denoting the optical density of each of the RGB channels. $A$ is a three-by-three absorption matrix, of which the rows indicate the different RGB channels and columns indicate the different stains. $C$ is a three-element vector representing the concentration of each stain. Once $A$ is determined, the color deconvolution scheme is applied according to

$$
C=A^{-1} \Delta .
$$

In our study, there are two principal stains, hematoxylin and KL1. The optical densities of the specific stains were obtained by an automatic singular value decomposition (SVD) method, based on which the absorption matrix $A$ was created following the process detailed in [39]. The color deconvolution was then performed to separate the hematoxylin and KL1 channels as shown in Fig. 4(b), (c).

\section{3) Cell nuclei segmentation}

To segment cells on histological images with high accuracy is a big challenge, since cells have high variance in size and shape. In addition, artifacts coming from the histological image preparation (tissue slice cutting and staining) further impede cell segmentation. In this paper, we implemented a seed based automatic and robust cell segmentation algorithm which is composed of two steps. The first step was to automatically locate the center of each cell using local intensity minimum and gradient information [26]. Cell centers detected in the first step were then used as initial positions for the second step for nucleus segmentation and nucleus contour extraction.

To determine the seed points, only the hematoxylin channel (Fig. 4(b)) extracted above by color deconvolution from the color image (Fig. 4(a)) was used, as including KL1 staining reduced contrast. The hematoxylin channel was converted into a gray scale field $I(x, y)$ (Fig. 4(d)). The image gradient was computed with the Sobel operator. Its direction is from the center of a nucleus to the outside. The gradient for each pixel was used to compute a voting area (Fig. 4(f)) denoting the most probable (candidate) nuclei region as follows: for each $\operatorname{pixel}(x, y)$, the "voting direction" $\alpha(x, y)$ is defined as

$$
\alpha=\frac{-\nabla I(x, y)}{\|\nabla I(x, y)\|}=-(\cos (\theta(x, y)), \sin (\theta(x, y))),
$$

where $\nabla I(x, y)$ is the gradient, $\|\nabla I(x, y)\|$ is the gradient magnitude, and $\theta$ is the angle of the gradient direction with respect to x-axis. The voting area $A_{\text {vote }}\left(x, y ; l_{\text {min }}, l_{\text {max }}, \beta\right)$ of each pixel is defined by an annular sector with its vertex at $(x, y)$ as illustrated in Fig. 4(f). Based on the approximated mean nuclei diameter $(5 \mu \mathrm{m}), l_{\min }$ was set to $1.25 \mu \mathrm{m}$, and $l_{\text {max }}$ was set to $3.75 \mu \mathrm{m}$. $\beta$ is the angular range and set to $30^{\circ}$. Those regions where voting regions of different pixels overlap are likely to be nuclear center regions (Fig. 4(f)). A region overcoming a certain threshold was considered as center region, whereby the threshold was chosen to ensure one center region per nucleus. We defined the nucleus seed point as centroid of the region. The final nuclei segmentation was obtained by an active contour method [41] using the detected nuclei seed points (Fig.4(g)).

\section{$B .3 d$ cell density estimation with an ellipsoid model}

The $3 \mathrm{~d}$ cell density is estimated in a two-step process. First, we analyze how $2 \mathrm{~d}$ and $3 \mathrm{~d}$ cell densities relate for a given cell type (cancer vs. non-cancer cells). Second, based on these relationships, the total $3 \mathrm{~d}$ cell density is computed combining the number of cancer and non-cancer cells (this will lead to (7) in Section IV.D). The first step is the focus of this section, while the results for the two steps are given in Sections IV B, D.

An iterative optimization method is proposed to estimate the nuclei distribution in $3 \mathrm{~d}$ using the $2 \mathrm{~d}$ nuclei features of a given cell type. Our method estimates a statistical distribution of cells in $3 \mathrm{~d}$ space but cannot give their true $3 \mathrm{~d}$ spatial distribution. 


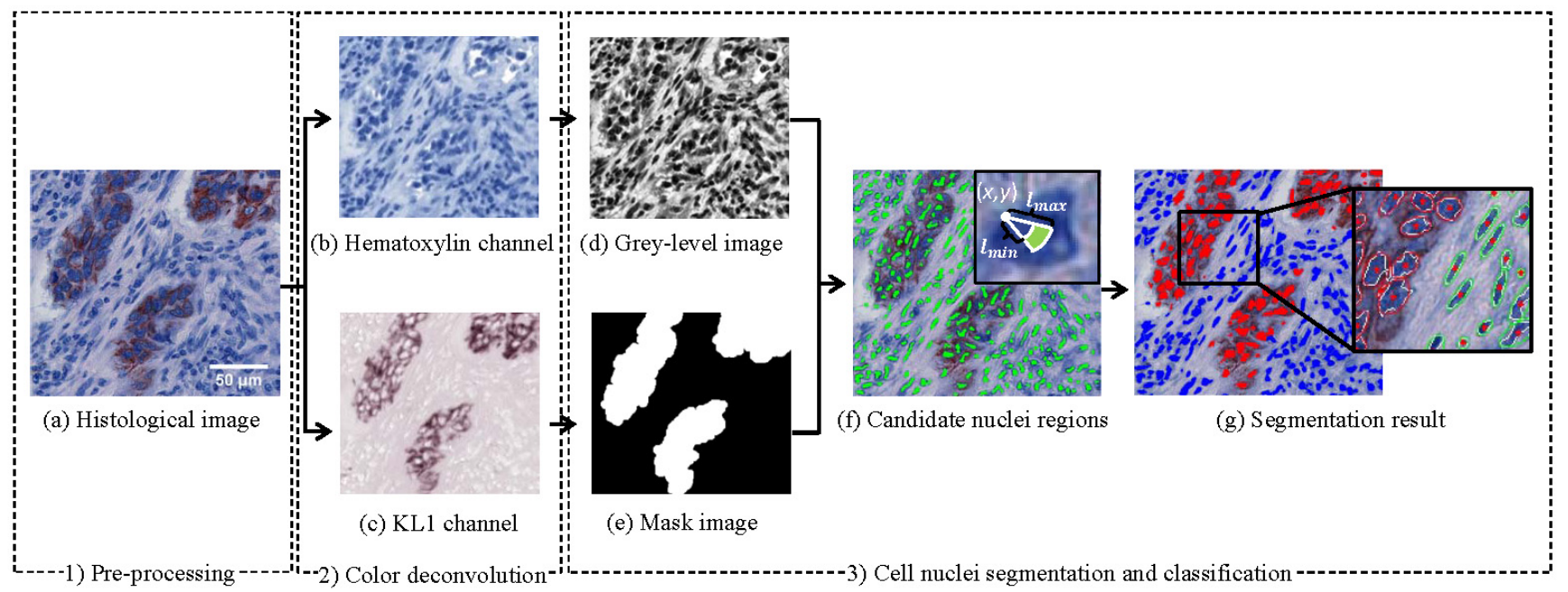

Fig. 4. Automatic cell nuclei detection: (a) histological color image was separated into (b) hematoxylin (cell nuclei in blue) and (c) KL1 (cancer cell sensitive regions in brown) channels; based on the grey level image (d) (converted from the hematoxylin channel) and the binary mask image (e) (extracted from the KL1 channel), candidate nuclei regions (green) (f) were obtained; the final segmentation result (g) including the cancer nuclei (red) and non-cancer nuclei (blue), the small window on the right shows the nuclei contours and center points inside the zoom region.

Fig. 5 illustrates the $3 \mathrm{~d}$ density estimation algorithm that will be applied in section IV.B.

Our method is based on the observation that the shape of a cell nucleus in $2 \mathrm{~d}$ is approximately elliptical [27], [28], [42]. Accordingly we assumed that nuclei in $3 \mathrm{~d}$ are ellipsoids [43], [44] (Fig. 5(a)).

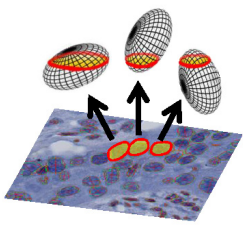

(a)

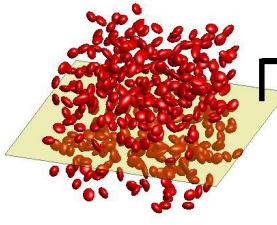

(b)

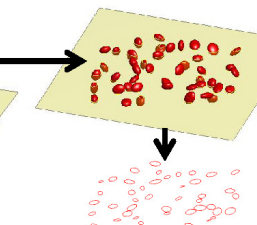

(c)
Fig. 5. Illustration of the conceptual idea underlying the $3 \mathrm{~d}$ cell density estimation: (a) The elliptic nuclei contours in the $2 \mathrm{~d}$ histological image ( 3 red circles) are assumed to represent cuts of $3 \mathrm{~d}$ ellipsoidal nuclei. (b) If the ellipsoids are distributed in a $3 \mathrm{~d}$ model tissue volume, (c) virtual cuts through the $3 \mathrm{~d}$ virtual tissue volume (yellow plane in (b) and (c)) should statistically reproduce the shape distribution of $2 \mathrm{~d}$ cross-sections from (a) indicated by the red cut contours in the bottom of (c) provided that the axis lengths and density of the ellipsoids are properly chosen.

The $2 \mathrm{~d}$ cell density and nuclei size distribution are calculated based on the $2 \mathrm{~d}$ nuclei segmentation results on the histological images. The $2 \mathrm{~d}$ contours of detected nuclei are fitted by ellipses with semi-major axis $a$ and semi-minor axis $b$. As nuclei contours are of different size, this leads to nuclei size distributions $f(a), h(b)$, respectively. We assume that the $2 \mathrm{~d}$ contours emerge from cross sections of ellipsoidal shaped nuclei in $3 \mathrm{~d}$ whereby the ellipsoids have semi-axes $a^{\prime}, b^{\prime}$ and $c^{\prime}$ (Fig. 5(a)). The axes are determined empirically requiring that: (i) the density of objects in virtual $2 d$ cross sections through a 3d volume (Fig. 5(b)), into which the ellipsoids were homogeneously distributed and isotropically oriented, shall reproduce the $2 \mathrm{~d}$ density in the data (Fig. 5(b),(c)); (ii) the distribution of semi-major and semi-minor axes in the virtual $2 \mathrm{~d}$ cross sections should reproduce the expectation values $\langle a\rangle,\langle b\rangle$ and the variances $\left\langle(\delta a)^{2}\right\rangle,\left\langle(\delta b)^{2}\right\rangle$ obtained from the data. For this an iterative procedure is used (Algorithm 1) where the ellipsoid axes are drawn from normal distributions:

$$
\left\{\begin{array}{c}
a^{\prime} \sim N\left(r_{\text {maj }}, \sigma_{\text {maj }}\right) \\
b^{\prime} \sim N\left(r_{\text {min }}, \sigma_{\text {min }}\right) \\
c^{\prime} \sim N\left(\sqrt{r_{\text {maj }} r_{\text {min }}}, \sqrt{\sigma_{\text {maj }} \sigma_{\text {min }}}\right) .
\end{array}\right.
$$

As starting values of the iteration, values $r_{\text {maj }}=\langle a\rangle, r_{\text {min }}=$ $\langle b\rangle, \sigma_{\text {maj }}=\left\langle(\delta a)^{2}\right\rangle$, and $\sigma_{\min }=\left\langle(\delta b)^{2}\right\rangle$ are used and stepwise adjusted until the upper conditions (i) and (ii) are fulfilled. Notice that this choice is neither unique, nor compulsory. For example, it neglects a possible correlation between major and minor axes in the $2 \mathrm{~d}$ section and in $3 \mathrm{~d}$ (through the nucleus volume for which we do not have data). However, we validated the algorithm with liver tissue for which we could reconstruct the $3 \mathrm{~d}$ volume data from optical sections of confocal laser scanning micrographs, and we verified the algorithm with synthetic data on elongated cell nuclei shapes, with both homogeneous and inhomogeneous distributions of cell nuclei (Supplementary ${ }^{1}-\mathrm{A}$ ).

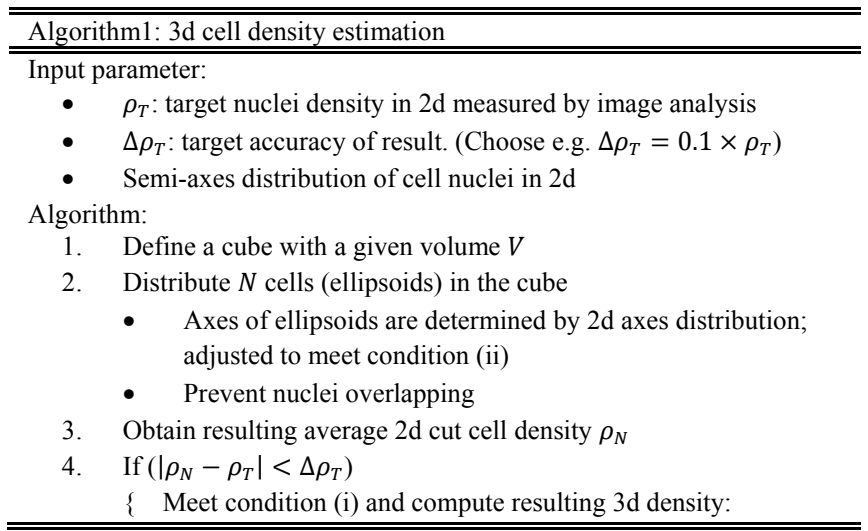

${ }^{1}$ Supplementary materials are available in the supplementary files /multimedia tab. 


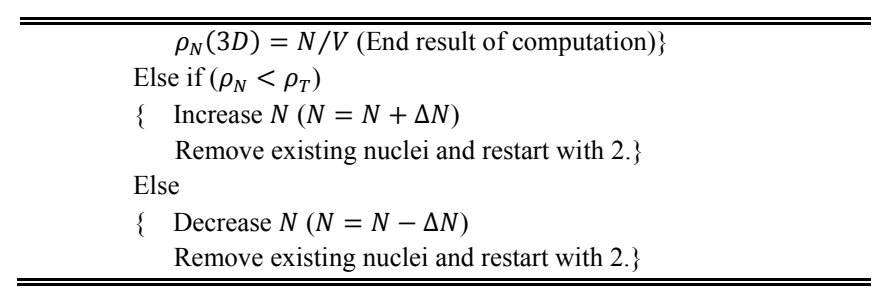

C. Cell number estimation by correlation between diffusion and cellularity

The tumor cell number was calculated based on tumor voxels' $\mathrm{D}$ values and the identified correlation between diffusion and cellularity. Fig. 6 illustrates the mapping from D value to local cell density: the high $\mathrm{D}$ value voxel has low cell density, while the low D voxel has high cell density. The correlation and cell number counting scheme are explained in detail in Section IV.

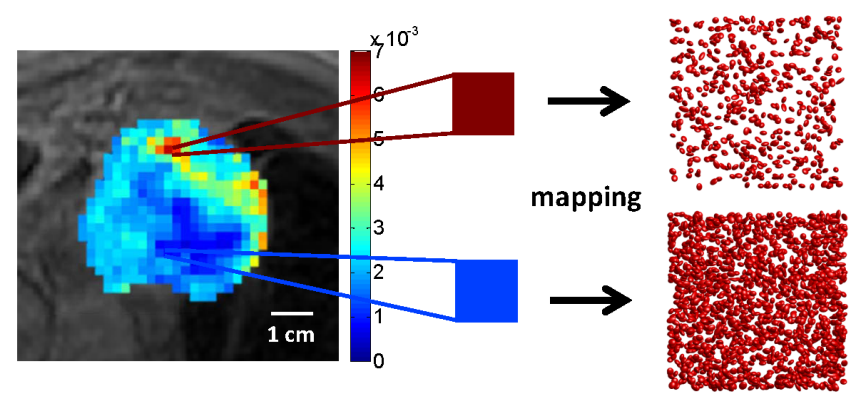

Fig. 6. Mapping from $\mathrm{D}$ value to local cell density. T1-MRI overlaid with the D parametric map of the selected tumor (left), the colorbar indicates the D values displayed in the tumor region; high $\mathrm{D}$ value corresponds to low cell density (sparsely distributed nuclei) while low $\mathrm{D}$ value corresponds to high cell density (densely distributed nuclei)

\section{ANALYSIS RESUlts}

\section{A. Cell segmentation and classification evaluation}

The proposed automatic cell segmentation algorithm performs high detection accuracy (Fig. 7); this was confirmed by the study pathologist whose evaluation served as 'diagnostic standard'. For this, we selected 20 representative histological images, and then compared the detected results by the proposed method with the manual results. First we validated the cell segmentation, and then among the detected cells, the cell classification in cancer versus non-cancer cells. The TP, TN, FP, and FN ( $T=$ true, $F=$ false, $N=$ negative, $P=$ positive) of cell segmentation are defined in Supplementary ${ }^{1}$-B. Fig. 7(a) shows the ROC graph for the cell segmentation (blue solid triangles) and classification performance of identifying cell types (red solid circles) for the 20 registered images (each represented by a symbol). The results indicate high sensitivity $(=T P /(T P+F N))$ and specificity $(=T N /(T N+F P))$ in all cases. Table I lists the corresponding mean values and standard deviations of sensitivity, specificity and accuracy $(=(T P+T N) /(T P+F P+F N+T N))$. The proposed algorithm has a high mean accuracy for cell segmentation and cancer cell vs. non-cancer cell classification (up to 0.963 ) illustrating that the algorithms can reliably detect and identify individual cells/nuclei in the histological sections. The analysis was later done on 10 original images: there seems to be no influence of the cutting procedure (spatial distortion) on the cell counts.

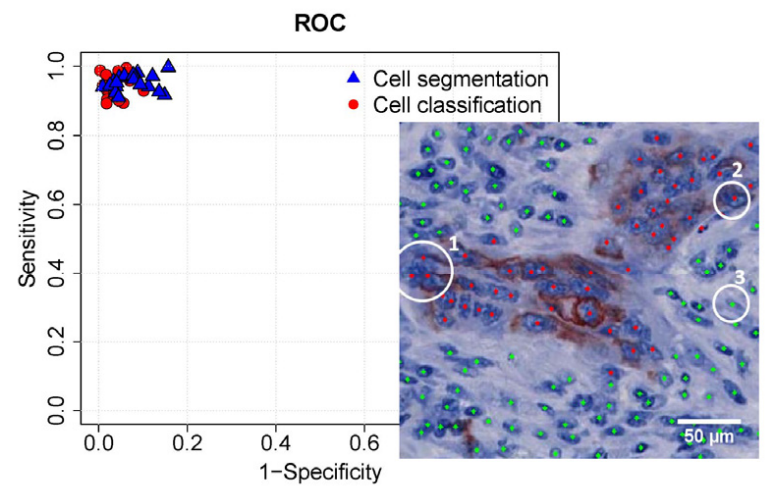

(a)

(b)

Fig. 7. Evaluation of cell segmentation and classification. (a) ROC graph for performance of cell segmentation (blue solid triangle) and classification (red solid circles). (b) One sample of the result image: tumor cell nuclei are stained in blue (Hematoxylin), the cancerous regions are stained in brown (KL1), the red points indicate the detected cancer cell nuclei center points, while green points indicate the non-cancer cell nuclei. The algorithm can reliably detect individual nuclei even in areas with high cell density. For example in circle 1 individual nuclei are recognized in areas with visually overlapping nuclear structures. In addition, individual cytokeratin positive tumor cells (circle 2) and stromal fibroblasts (circle 3 ) can be detected.

TABLE I

EVALUATION OF THE CELl SEGMENTATION AND CLASSIFICATION ON 20 SELECTED REGISTERED AND 10 ORIGINAL HISTOLOGICAL IMAGES $(0.47 \mathrm{~mm} \times$ $0.47 \mathrm{~mm}$ ): FROM LEFT TO RIGHT EXPRESSED AS MEAN \pm THE STANDARD DEVIATION, SENSITIVITY, SPECIFICITY, AND ACCURACY.

\begin{tabular}{|l|l|l|l|}
\hline Performance & Sensitivity & Specificity & Accuracy \\
\hline \multicolumn{4}{|c|}{ Results on registered images } \\
\hline Cell segmentation & $0.954 \pm 0.026$ & $0.946 \pm 0.042$ & $0.953 \pm 0.017$ \\
\hline Classification & $0.947 \pm 0.030$ & $0.968 \pm 0.022$ & $0.963 \pm 0.017$ \\
\hline \multicolumn{4}{|c|}{ Results on original images } \\
\hline Cell segmentation & $0.950 \pm 0.023$ & $0.943 \pm 0.029$ & $0.947 \pm 0.016$ \\
\hline Classification & $0.956 \pm 0.056$ & $0.952 \pm 0.026$ & $0.954 \pm 0.032$ \\
\hline
\end{tabular}

B. 3 d cell density estimation: cancer vs. non-cancer cells

First, in order to investigate the $3 \mathrm{~d}$ cell density, we fitted ellipses to the segmented nuclei in the histological images and computed the semi-major and semi-minor axes in the 2D slices for all blocks obtaining the distributions in Fig. 8. The pooling from all slices is justified, as not only the intra-block but also the inter-block variability of the ellipse axes is small (see Supplementary ${ }^{1}-\mathrm{C}$ ). The average values and the variances of semi-axes over all the blocks were used for computation of the parameters $a^{\prime}, b^{\prime}$ and $c^{\prime}$ as explained in Section III.B.

To probe the relationship between $2 \mathrm{~d}$ and $3 \mathrm{~d}$ cell densities for the selected tumor, we mimicked the cell distribution in the tumor volume and the tissue cuts. We assumed the $2 \mathrm{~d}$ cell density for each cell type (cancer or non-cancer cells) increasing from $1 \mathrm{cell} / \mathrm{mm}^{2}$ to $6000 \mathrm{cell} / \mathrm{mm}^{2}$ by step 1 cell $/ \mathrm{mm}^{2}$. For each of the given $2 \mathrm{~d}$ density value, we estimated the corresponding $3 \mathrm{~d}$ cell density according to Algorithm 1 . We defined a cube $\mathrm{V}$ with edge length of $1 \mathrm{~mm}$, and then distributed a number of ellipsoids in $\mathrm{V}$ in random locations and orientations, according to distributions $a^{\prime}, b^{\prime}$ and $c^{\prime}$. The estimated $2 \mathrm{~d}$ cell density is defined as the number of ellipsoid sections on the virtual cutting plane (Fig. 5(c)) divided by the area of the cutting cross-section. The estimated $3 \mathrm{~d}$ cell density equals the number of ellipsoids divided by the volume $\mathrm{V}$. The 
estimation was repeated for 10 times for each of the given $2 \mathrm{~d}$ cell density. Finally, by plotting the estimated $3 \mathrm{~d}$ cell density against the given $2 \mathrm{~d}$ cell density for each estimation, a linear relationship was indicated for each cell type, $\rho_{3 D}^{k}=m^{k} \rho_{2 D}^{k}$ with $k \in\{c, n c\}$ for cancer and non-cancer cells, respectively (Fig. 9, showing the data points with $3 \mathrm{~d}$ density ranging from about 1000 to $320,000 \mathrm{cell} / \mathrm{mm}^{3}$ ). The least-square regression lines were drawn for both cancer and non-cancer cases. For cancer cells the slope $m^{c}=96 / \mathrm{mm}$ (goodness of fit: $\mathrm{R}^{2}=$ $0.9997)$, while for non-cancer cells $m^{n c}=115 / \mathrm{mm}\left(\mathrm{R}^{2}=\right.$ 0.9996).
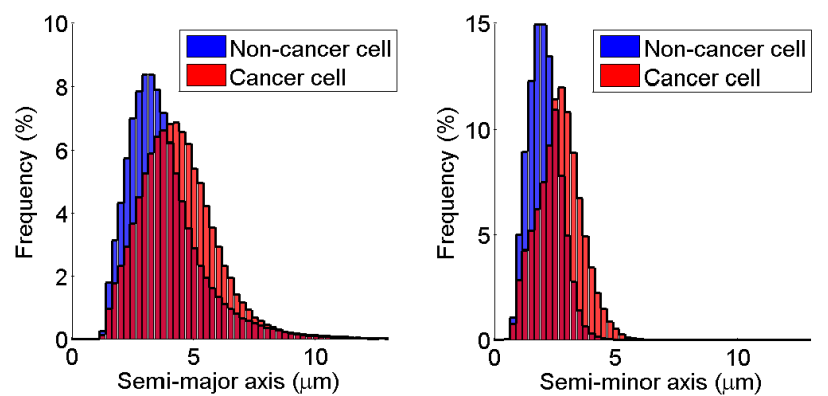

Fig. 8. Elliptical semi-major (left) and semi-minor (right) axes distributions in $2 \mathrm{~d}$ histological images for cancer cells (in red) and non-cancer cells (in blue). For cancer cells, the average semi-major axis is $4.38 \mu \mathrm{m}(S D=1.62 \mu \mathrm{m})$; the average semi-minor axis is $2.68 \mu \mathrm{m}(S D=0.9 \mu \mathrm{m})$. For non-cancer cells, the average semi-major axis is $3.85 \mu \mathrm{m}(S D=1.92 \mu \mathrm{m})$; the average semi-minor axis is $2.05 \mu \mathrm{m}(S D=0.66 \mu \mathrm{m})$

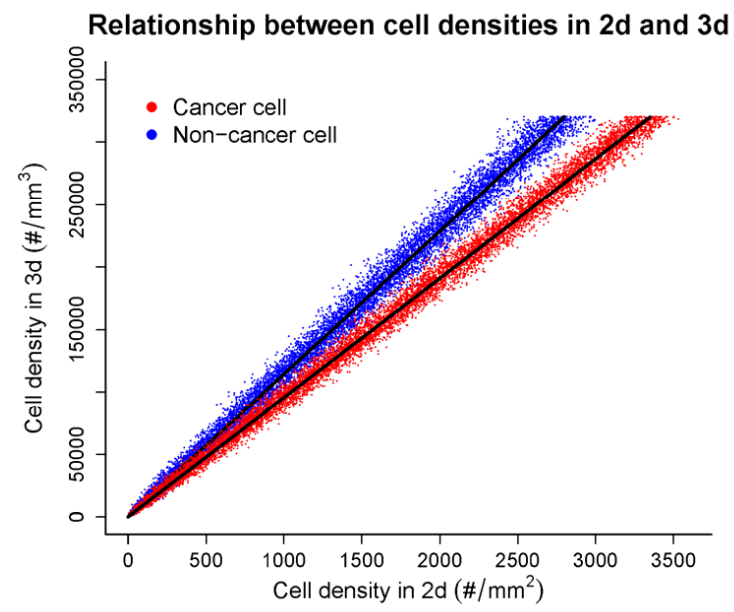

Fig. 9. Linear relationship between $2 \mathrm{~d}$ and $3 \mathrm{~d}$ cell densities for both cancer and non-cancer cells. The slope of the line depends on the cell size (the semi-major and semi-minor axes of cell nuclei), the larger the cell (cancer cells) the lower the slope.

\section{Tumor homogeneity analysis: cell density distribution and cancer region fraction}

The representative tumor samples (Fig. 10(a)) are selected for the study of the tumor homogeneity and cellularity. The $2 \mathrm{~d}$ cell density was tested for significant differences among the selected tumor samples (S1-S7) with the Kruskal-Wallis test [45]. For each tumor sample, 50 histological slides uniformly distributed through the tumor block were selected for the statistical test. The null hypothesis (HO) is that there is no significant difference of cell density among tumor samples (the tumor is homogeneous). The hypothesis was rejected, as the difference is statistically significant ( $\mathrm{p} \ll 0.001)$. The tumor is thus heterogeneous.

The cancer region area fraction of tumor samples is presented by plotting the cancer (KL1 positive) region area against the non-cancer (non-KL1 positive) region area as shown in Fig. 10(b). The tumor sample S3, which is the closest to the necrosis region, has the highest cancer region fraction, while the tumor samples with lung tissue $\mathrm{S} 1$ and with fat tissue S7 have lower cancer region fractions. In case of a homogeneous tumor, all points on Fig. 10(b) would be on one straight line.

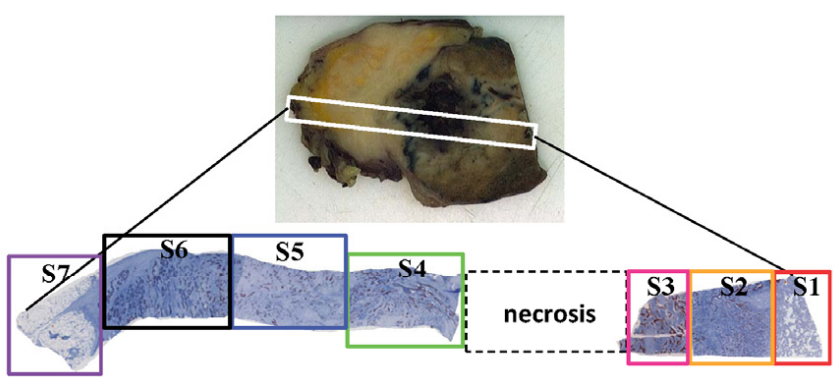

(a)

Cancer vs. Non-cancer positive regions in tumor

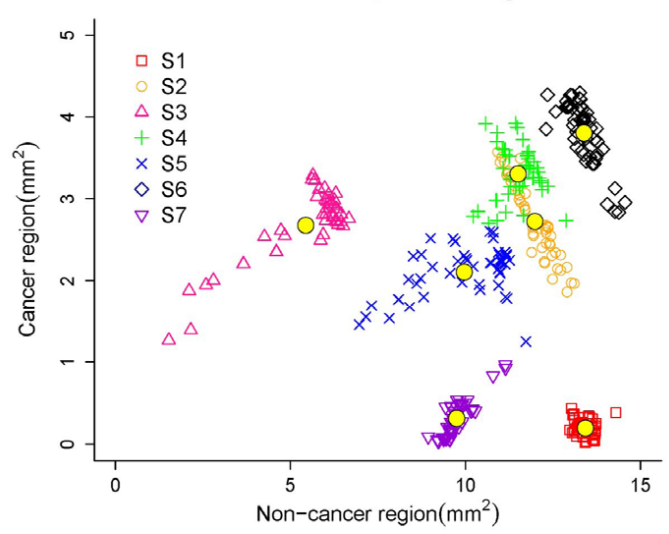

(b)

Fig. 10. Heterogeneity of the tumor: (a) overview of the tumor samples (S1-S7); (b) comparison of the cancer region fraction between different tumor samples by plotting the cancerous (KL1 positive) region area against the non-cancerous (non-KL1) region area, for 50 slides per tumor block. The yellow solid point denotes the average for the respective tumor sample.

\section{Correlation between $D$ value and cell density}

To investigate the correlation between diffusion and cellularity, we performed quantitative analyses of $\mathrm{D}$ values and cell densities of the selected tumor samples (Fig. 10(a)). Table II lists the D values of different tumor samples, which were calculated by performing IVIM estimation on the ROIs of the tumor samples on DWI (Fig. 11). The values of the fitted $\mathrm{f}$ and $D^{*}$ are given in Supplementary ${ }^{1}-\mathrm{D}$. The average goodness of fit $\left(\mathrm{R}^{2}\right)$ of (1) to our data is 0.91 , and the corresponding standard deviation is 0.06 . The $2 \mathrm{~d}$ cell density (cancer and non-cancer cells together) of each tumor sample is the mean cell density of the 50 histological slides uniformly distributed through the tumor block. 
TABLE II

D VALUES (MEAN AND STANDARD DEVIATION (SD) IN $\left.\left(10^{-3} \mathrm{~mm}^{2} / \mathrm{s}\right)\right)$ OF THE DIFFERENT TUMOR SAMPLES DEFINED IN Fig. 10(a)

\begin{tabular}{|c|c|c|c|c|c|c|c|}
\hline Blocks & S1 & S2 & S3 & S4 & S5 & S6 & S7 \\
\hline D & 2.985 & 1.632 & 1.095 & 1.809 & 1.963 & 2.064 & 2.404 \\
\hline $\begin{array}{c}\text { SD of } \\
\text { D }\end{array}$ & 0.328 & 0.122 & 0.079 & 0.027 & 0.018 & 0.050 & 0.037 \\
\hline
\end{tabular}

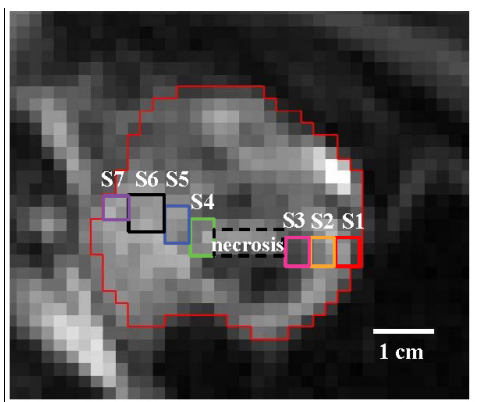

Fig. 11. ROIs on DWI corresponding to the seven tumor samples (Fig. 10(a)). The shapes of the histological specimens were translated back to the DWI data by manual co-registration. Necrosis has been identified from T1-CEFS-MRI (Fig. 2(b)).

Drawing the $2 \mathrm{~d}$ cell densities of tumor samples against the corresponding $\mathrm{D}$ values exhibits a strong negative linear correlation (Fig. 12). The Pearson product-moment correlation test was performed to evaluate the linear correlation. The tumor sample S3 is next to the necrosis region (Fig. 10(a)), which has the highest tumor cell density with lowest diffusion coefficient. $\mathrm{S} 1$ includes part of healthy lung tissue. Because of the alveoli of lung tissue, there are cell-free regions in $\mathrm{S} 1$; this reduces the cell density and allows high mobility of water molecules (high diffusion). S7 is located at the tumor boundary where it has grown into the pericardial fat. A typical fat cell is quite big. For example, the white fat cell is $0.1 \mathrm{~mm}$ in diameter [46], which is the major form of adipose tissue in mammals. Therefore, fat tissue has low cellularity. Besides, we found a negative correlation for the $2 \mathrm{~d}$ nuclei area fraction (total area of nuclei sections divided by area of tissue $\left(\mathrm{A}^{\text {tissue }}\right)$ for each sample slide) and the $2 \mathrm{~d}$ cell area fraction (total area of cell sections divided by $A^{\text {tissue }}$ ) vs. the $D$ value (see Supplementary ${ }^{1}-E$ and $-F$ ).

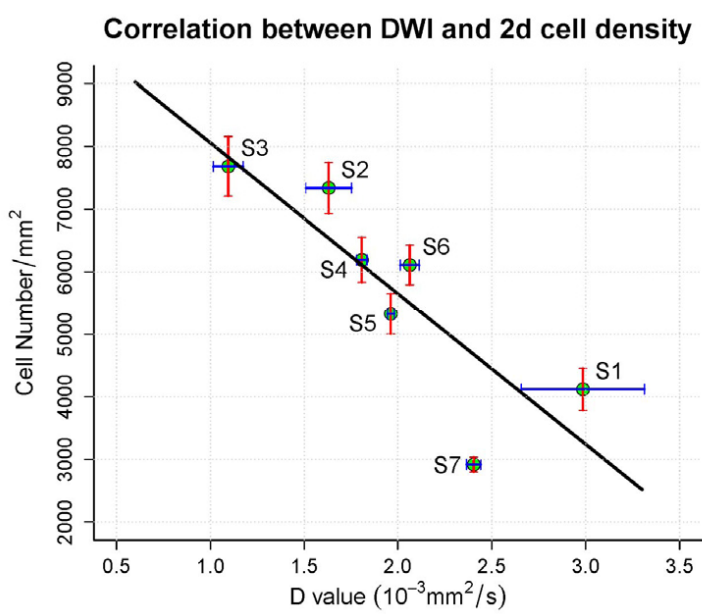

Fig. 12. Correlation between $\mathrm{D}$ value and $2 \mathrm{~d}$ cell density. The Pearson correlation coefficient $(\gamma)$ is -0.84 , representing a strong negative linear correlation. High diffusion in tissue predicts low cell densities (and vice versa). The line represents a least mean-square fit of the data.
A negative linear correlation is also observed between $3 \mathrm{~d}$ cell density and $\mathrm{D}$ value (Fig. 13). The $3 \mathrm{~d}$ cell density $\rho_{3 d}$ was computed based on the cancer region fraction $\phi^{c}$ and the intrinsic cell density slope factor $m^{c}$ (for cancer cell) and $m^{n c}$ (for non-cancer cell) from $2 \mathrm{~d}$ to $3 \mathrm{~d}$ (Fig. 9):

$$
\rho_{3 \mathrm{~d}}=\phi^{c} * \rho_{2 d}^{c} * m^{c}+\left(1-\phi^{c}\right) * \rho_{2 d}^{n c} * m^{n c},
$$

where $\phi^{c}$ is the ratio between the cancer area (KL1 stained tissue area) and the tumor area (total tissue area). The intrinsic cancer cell density $\rho_{2 d}^{c}$ equals the number of cancer cells divided by the cancer region (KL1) area, and the intrinsic non-cancer cell density $\rho_{2 d}^{n c}$ equals the number of non-cancer cells divided by the non-cancer region area (non-KL1).

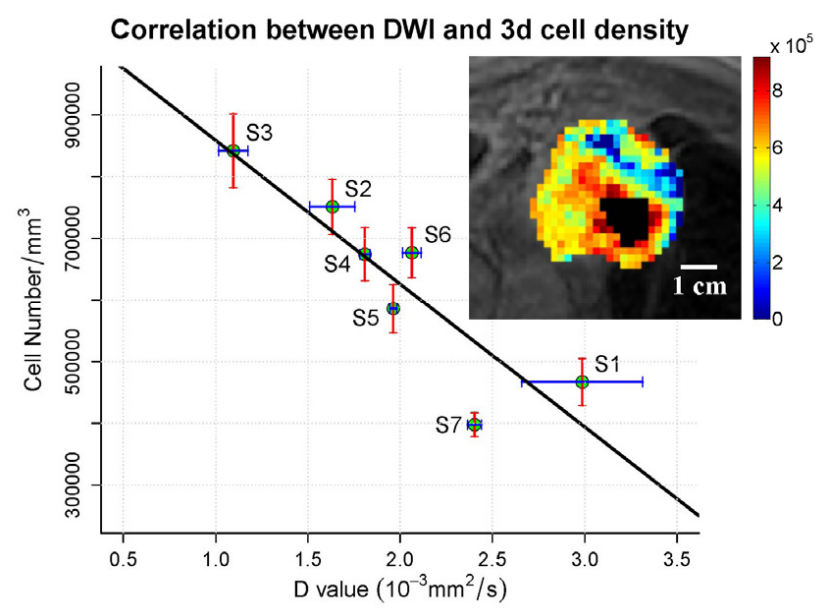

Fig. 13. Correlation between $\mathrm{D}$ value and $3 \mathrm{~d}$ cell density. The Pearson correlation coefficient $(\gamma)$ is -0.89 , which represents a strong linear negative correlation. The line represents a least mean-square fit $\rho_{3 d}=g(\mathrm{D})$. Inset: cell density map of tumor section at DWI resolution excluding the necrotic region (in black), which is identified in T1-weighted MRI (compare Fig. 11).

\section{E. Tumor cell count estimation}

Given the D value of each voxel in the tumor DWI data, the $3 \mathrm{~d}$ cell density corresponding to each voxel is obtained by the mapping from the $\mathrm{D}$ value to the local cell density. We exclude the necrotic volume in the calculation of cell number. The cell number of the whole tumor is the sum of the local cell numbers of vital tumor voxel volumes (excluding necrosis),

$$
N_{\text {tumor }}=\sum_{i} N(i)=\sum_{i} \rho_{3 \mathrm{~d}}(i) \times v(i)=\sum_{i} g(D(i)) \times v(i) \text {. }
$$

Here $N(i)$ is the cell number of voxel $i, \rho_{3 d}(i)$ is the voxel $3 \mathrm{~d}$ cell density, and $v(i)$ is the voxel volume, $v(\mathrm{i})=2.1 \mathrm{~mm} \times$ $2.1 \mathrm{~mm} \times 6 \mathrm{~mm}=26.46 \mathrm{~mm}^{3}$. The linear mapping $g(D(i))$ is defined above (see Fig. 13). The total cell number of the lung tumor in this study is estimated as $2.57 \times 10^{10}$.

The average cell density of the whole tumor is $5.9 \times$ $10^{5} \mathrm{cell} / \mathrm{mm}^{3}$ computed by dividing the total cell number by the vital tumor volume. It is comparable to the average density of the tumor samples $(\mathrm{S} 1-\mathrm{S} 7) 6.28 \times 10^{5} \mathrm{cell} / \mathrm{mm}^{3}$. If we compute the total tumor cell number by multiplying the vital tumor volume in the T1-weighted MRI data with the average cell density of the tumor samples, the cell count is $4.52 \times$ $10^{4} \mathrm{~mm}^{3} \times 6.28 \times 10^{5} \mathrm{cell} / \mathrm{mm}^{3}=2.84 \times 10^{10}$.

T1-weighted MRI seems to lead to a larger total tumor volume than DWI $\left(4.35 \times 10^{4} \mathrm{~mm}^{3}\right)$, but the difference between tumor cell load estimations through both methods is not large. 


\section{DiscusSiOn AND CONCLUSION}

Exemplified for a NSCLC patient, we here present a new pipeline for the estimation of tumor cell load in solid tumors based on the fusion of image processing techniques. As a result, tumor cell numbers may directly be inferred from the non-invasive DWI data, which is routinely available for cancer patients. The main novelties include: adaptive cell nuclei segmentation; model-based $3 \mathrm{~d}$ cell nuclei density estimation from $2 \mathrm{~d}$ cell nuclei data; cell number calculation based on the correlation between diffusion coefficient (D value) obtained from the DWI data and $3 \mathrm{~d}$ cell densities. The inference of $3 \mathrm{~d}$ nuclei density from $2 \mathrm{~d}$ sections is crucial to estimate tumor cell load in each voxel of the DWI as well as the whole tumor. Despite the algorithm is simple and approaches nuclei shapes by simple ellipsoids, it could be validated using confocal micrographs of liver tissue to give reliable values, and verified with synthetic data (Supplementary $\left.{ }^{1}-\mathrm{A}\right)$. The inference algorithm could further be refined by taking into account the nucleus volume distribution, permitting to identify correlations between the ellipsoid axes. However, we do not expect major differences for the questions studied in this paper.

The representative tumor samples were separated from the tumor mass after surgical resection. The automatic histology evaluation was successfully validated by a pathologist, showing high accuracy of the algorithm. The radiologist localized the representative tumor sample, defined the tumor borders in the DWI, and calculated for each tumor sample its corresponding $\mathrm{D}$ value by averaging over all voxels belonging to the respective sample. Knowing the $\mathrm{D}$ values of the whole tumor and the relationship between the $\mathrm{D}$ values and cell densities, the total tumor cell number was finally obtained.

For the histological study of the selected tumor, the digital cell segmentation successfully distinguished cancer cells from non-cancer cells, and their respective fractions revealed tumor heterogeneity. The segmentation and classification perform very well (Table I), but might be further improved by applying an image-specific color deconvolution method [47]. Heterogeneity was also indicated by the inter tumor samples cell density standard deviation $\mathrm{SD}_{\text {inter }}=1700 \mathrm{cell} / \mathrm{mm}^{2}$ having been more than five times the standard deviation of the intra sample density $\mathrm{SD}_{\text {intra }}=330 \mathrm{cell} / \mathrm{mm}^{2}$. The tumor region close to the necrosis part has much higher cell densities than the region merged with the lung tissue or pericardial fat. In DWI, a high D value was obtained in tumor regions with low cell density, while low D value was obtained for high cellularity region. The $\mathrm{D}$ value of the tumor samples ranges between 1.09 and $2.98 \times 10^{-3} \mathrm{~mm}^{2} / \mathrm{s}$ with mean value $1.99 \times 10^{-3} \mathrm{~mm}^{2} / \mathrm{s}$. The $\mathrm{D}$ value differences in DWI indicate the variation of local cell density of tumor samples, which ranges between $2917 \mathrm{cell} / \mathrm{mm}^{2}$ and $7684 \mathrm{cell} / \mathrm{mm}^{2}$. The model-based computed $3 \mathrm{~d}$ tumor densities varying within the tumor by a factor of about 2 were within the range denoted in [48]. Moreover this spatial organization of cell densities in a tumor is coherent with classical solid tumor descriptions [49]. DWI may thus provide a non-invasive indicator of such microscale structure.

Our study may help to refine and adjust the patient-specific development of therapeutic strategies. This holds true for conventional cytostatic drugs (e.g. platin based regimens) and for highly selective enzyme inhibitors (e.g. the epidermal growth factor inhibitor erlotinib), which are currently used in the treatment of lung cancer. In addition, this algorithm may be beneficial for the personalized adjustment of novel therapeutic approaches such as bi-specific monoclonal antibodies (BiTE) or immunotherapeutic therapies. Here the knowledge of cell numbers (cancer and non-cancer), as well as vessel density (which is key for pharmacokinetics) may allow the precise fitting of drug concentrations and application modes in order to reduce side effects and to increase the drugs anti-tumor efficiency. Thus, differences in the diffusion coefficient (D value) could not only aid the diagnosis but also guide the therapeutic intervention. Our procedure, tested for one selected NSCLC patient, might straightforwardly be applied to other cancer types, for which specific biomarkers for cancer cells exist. Stains used in tissue preparation other than KL1, will also be successful as long as individual cell types (cancer and non-cancer) can be identified based on the morphology or epitope-based approaches.

Potential errors of our study may include a mis-fusion of the MRI imaging and pathology processing of tissue specimens. For example, the shrinkage of tissue samples after formalin fixation [50] (around 10\% [51]), may affect the tumor size measurement and must be considered in the future for tumor volume and $2 \mathrm{~d} / 3 \mathrm{~d}$ cell density estimations. The tissue deformation caused by cutting artifacts may be corrected by image registration [38], however, as was shown in this paper, the registration step is not compulsory (see Table I). Limitations of the anatomical MRI planning, such as tilts of less than $5^{\circ}$ of the DWI plane vs. the histology plane, increase the difficulty of the co-registration of the histological tumor slide and MRI data; however, by slight variation of the included DWI voxels we verified the robustness of the obtained $D$ values. The reproducibility of DWI in lung tumor is still under investigation, but the DWI data is reproducible in many organs and different tumor types [52]-[54].

Concerning the time-consumption of the different steps: the histological data generation is the most tedious part, whereas its automated image analysis does not require much man-time (and even computational time: 1 slide takes about $\sim 1 \mathrm{~h}$ on a 10 -core computer). The DWI data acquisition and parameter estimation are both much shorter than for histology ( 1h).

A further question remains of how this finding might be applied in the clinics. If the relation of $\mathrm{D}$ value and $3 \mathrm{~d}$ density is sufficiently general it could be applied to different tissues. However, as the D value reflects the sum of interactions with many obstacles, besides cell membranes such as macromolecules, fibers, desmoplastic reaction and vascularization, differences would be expected for different tissues, even if the qualitative behavior should not change as indicated by the negative correlation between cell density and $\mathrm{ADC}$ ( $\mathrm{D}$ value in our notion) observed in different tumors and patients in literature [55]. To further test its validity, the proposed method must be tested on patients with different subtypes of NSCLC tumors (e.g. squamous cell carcinoma and adenocarcinoma) to study how far the relation changes. For application in the clinics, one solution, even if labor-intense, would therefore be to systematically explore the correlation between cell density and D value for different tumor types (including origin at different organs) to obtain calibration 
curves that could be used for patients after identification of their tumor subtype. As an alternative applicable approach in the clinics, latest results indicate that the relation between D value and cell density might be inferred from biopsies, that in case of NSCLC patients are routinely taken for diagnosis guided by non-invasive imaging techniques. The biopsies should be sampled from regions of different $\mathrm{D}$-values.

\section{APPENDIX}

\section{A. Surgery protocol}

The study protocol was approved by the local Ethics Committee of the University of Heidelberg (approval number $\mathrm{S}-249 / 2010)$. In our study, the tumor tissue $(6.5 \mathrm{~cm}$ in diameter) was taken from a 72 year old patient with a NSCLC that was treated with an upper left lobe en-block resection including pericardial and mediastinal invaded parts (Fig. 1). Initial histology had been sampled by means of trans bronchial biopsy. The patient tumor, which was poorly differentiated squamous cell carcinoma, was classified as pT4 pN0 (0/27) pL2 G3 R2 (details given in Supplementary ${ }^{1}-\mathrm{G}$ ) according to TNM cancer staging system [56].

\section{ACKNOWLEDGMENT}

We thank Johannes Lotz and Janine Olesch for providing us with the registered images ([38]). We also thank the reviewers for their useful comments.

\section{REFERENCES}

J. J. Tomaszewski, R. G. Uzzo, and M. C. Smaldone, "Heterogeneity and renal mass biopsy: a review of its role and reliability.," Cancer Biol. Med., vol. 11, no. 3, pp. 162-172, 2014.

A. R. Padhani, G. Liu, D. M. Koh, T. L. Chenevert, H. C. Thoeny, T. Takahara, A. Dzik-Jurasz, B. D. Ross, M. Van Cauteren, D. Collins, D. A. Hammoud, G. J. Rustin, B. Taouli, and P. L. Choyke, "Diffusion-weighted magnetic resonance imaging as a cancer biomarker: consensus and recommendations," Neoplasia, vol. 11, no. 2, pp. 102-125, 2009 .

R. J. Theilmann, R. Borders, T. P. Trouard, G. Xia, E. Outwater, J. Ranger-Moore, R. J. Gillies, and A. Stopeck, "Changes in Water Mobility Measured by Diffusion MRI Predict Response of Metastatic Breast Cancer to Chemotherapy," Neoplasia, vol. 6, no. 6, pp. 831837, 2004.

[4] M. D. Pickles, P. Gibbs, M. Lowry, and L. W. Turnbull, "Diffusion changes precede size reduction in neoadjuvant treatment of breast cancer," Magn. Reson. Imaging, vol. 24, no. 7, pp. 843-847, 2006.

P. A. Hein, C. Kremser, W. Judmaier, J. Griebel, K. P. Pfeiffer, A Kreczy, E. B. Hug, P. Lukas, and A. F. DeVries, "Diffusion-weighted magnetic resonance imaging for monitoring diffusion changes in rectal carcinoma during combined, preoperative chemoradiation: Preliminary results of a prospective study," Eur. J. Radiol., vol. 45, no. 3, pp. 214-222, 2003.

D. M. Koh and D. J. Collins, "Diffusion-weighted MRI in the body: Applications and challenges in oncology," Am. J. Roentgenol., vol. 188, no. 6, pp. 1622-1635, 2007.

C. Reischauer, J. M. Froehlich, M. Pless, C. A. Binkert, D.-M. Koh, and A. Gutzeit, "Early treatment response in non-small cell lung cancer patients using diffusion-weighted imaging and functional diffusion maps--a feasibility study.," PLoS One, vol. 9, no. 10, p. e108052, 2014

D. Le Bihan, "Apparent diffusion coefficient and beyond: what diffusion MR imaging can tell us about tissue structure.," Radiology, vol. 268, no. 2, pp. 318-22, 2013. Functional Imaging in Oncology: Clinical Applications - Volume 2. Springer-Verlag Berlin Heidelberg, 2014.

[10] D. Le Bihan, E. Breton, D. Lallemand, M.-L. Aubin, J. Vignaud, and M. Laval-Jeantet, "Separation of diffusion and perfusion in intravoxel incoherent motion MR imaging," Radiology, vol. 168, no. 2, pp. 497-505, 1988.

[11] P. Marcon, K. Bartusek, and M. Cap, "Sensitivity of the Diffusion Coefficients Measurement to Gradient Field Changes," in PIERS Proceedings, 2011, pp. 80-83.

[12] K. Kono, Y. Inoue, K. Nakayama, M. Shakudo, M. Morino, K. Ohata, K. Wakasa, and R. Yamada, "The role of diffusion-weighted imaging in patients with brain tumors.", AJNR. Am. J. Neuroradiol., vol. 22, no. 6, pp. 1081-8, 2001

[13] Y. Hayashida, T. Hirai, S. Morishita, M. Kitajima, R. Murakami, Y. Korogi, K. Makino, H. Nakamura, I. Ikushima, M. Yamura, M. Kochi, J. Kuratsu, and Y. Yamashita, "Diffusion-weighted imaging of metastatic brain tumors: comparison with histologic type and tumor cellularity.," AJNR. Am. J. Neuroradiol., vol. 27, no. 7, pp. 1419-25, Aug. 2006.

[14] H. Lyng, O. Haraldseth, and E. K. Rofstad, "Measurement of Cell Density and Necrotic Fraction in Human Melanoma Xenografts by Diffusion Weighted Magnetic Resonance Imaging," Magn. Reson. Med., vol. 43, no. 6, pp. 828-836, 2000.

[15] A. W. Anderson, J. Xie, J. Pizzonia, R. A. Bronen, D. D. Spencer, and J. C. Gore, "Effects of cell volume fraction changes on apparent diffusion in human cells," Magn. Reson. Imaging, vol. 18, no. 6, pp. 689-695, 2000

[16] D. T. Ginat, R. Mangla, G. Yeaney, M. Johnson, and S. Ekholm, "Diffusion-weighted imaging for differentiating benign from malignant skull lesions and correlation with cell density," Am. J. Roentgenol., vol. 198, no. 6, pp. 597-601, 2012.

[17] K. M. Schmainda, "Diffusion-weighted MRI as a biomarker for treatment response in glioma," CNS Oncol., vol. 1, no. 2, pp. 169180, 2012.

[18] N. C. Atuegwu, D. C. Colvin, M. E. Loveless, L. Xu, J. C. Gore, and T. E. Yankeelov, "Incorporation of diffusion-weighted magnetic resonance imaging data into a simple mathematical model of tumor growth.," Phys. Med. Biol., vol. 57, no. 1, pp. 225-40, Jan. 2012.

[19] J. A. Weis, M. I. Miga, L. R. Arlinghaus, X. Li, A. B. Chakravarthy, V. Abramson, J. Farley, and T. E. Yankeelov, "A mechanically coupled reaction-diffusion model for predicting the response of breast tumors to neoadjuvant chemotherapy.," Phys. Med. Biol., vol. 58, no. 17, pp. 5851-66, Sep. 2013.

[20] J. A. Weis, M. I. Miga, L. R. Arlinghaus, X. Li, V. Abramson, A. B. Chakravarthy, P. Pendyala, and T. E. Yankeelov, "Predicting the Response of Breast Cancer to Neoadjuvant Therapy Using a Mechanically Coupled Reaction-Diffusion Model," Cancer Res., no. 615, 2015.

[21] D. A. Hormuth, J. A. Weis, S. L. Barnes, M. I. Miga, E. C. Rericha, V. Quaranta, and T. E. Yankeelov, "Predicting in vivo glioma growth with the reaction diffusion equation constrained by quantitative magnetic resonance imaging data.," Phys. Biol., vol. 12, no. 4, 2015.

M. Iima, O. Reynaud, T. Tsurugizawa, L. Ciobanu, J.-R. Li, F. Geffroy, B. Djemai, M. Umehana, and D. Le Bihan, "Characterization of glioma microcirculation and tissue features using intravoxel incoherent motion magnetic resonance imaging in a rat brain model.," Invest. Radiol., vol. 49, no. 7, pp. 485-90, 2014.

[23] A. M. Lemke, F. B. Laun, M. M. Klau, T. J. Re, D. Simon, S. Delorme, L. R. Schad, and B. Stieltjes, "Differentiation of Pancreas Carcinoma From Healthy Pancreatic," Invest. Radiol., vol. 44, no. 12, pp. 769-775, Dec. 2009.

[24] M. Veta, J. P. W. Pluim, P. J. van Diest, and M. A. Viergever, "Breast cancer histopathology image analysis: a review.," IEEE Trans. 
Biomed. Eng., vol. 61, no. 5, pp. 1400-11, May 2014.

E. Cosatto, M. Miller, H. P. Graf, and J. S. Meyer, "Grading nuclear pleomorphism on histological micrographs," in 2008 19th International Conference on Pattern Recognition, 2008, pp. 1-4

Qi, F. Xing, D. J. Foran, and L. Yang, "Robust segmentation of overlapping cells in histopathology specimens using parallel seed detection and repulsive level set.," IEEE Trans. Biomed. Eng., vol 59, no. 3, pp. 754-65, Mar. 2012.

[27] M. Veta, P. J. van Diest, R. Kornegoor, A. Huisman, M. A Viergever, and J. P. W. Pluim, "Automatic Nuclei Segmentation in H\&E Stained Breast Cancer Histopathology Images," PLoS One, vol. 8, no. 7, p. e70221, Jul. 2013.

[28] H. Xu, C. Lu, and M. Mandal, "An Efficient Technique for Nuclei Segmentation in Histopathological Images based on Morphological Reconstructions and Region Adaptive Threshold," IEEE J. Biomed. Heal. Informatics, vol. 18, no. 5, pp. 1729-1741, 2014.

[29] S. Wienert, D. Heim, K. Saeger, A. Stenzinger, M. Beil, P. Hufnagl M. Dietel, C. Denkert, and F. Klauschen, "Detection and segmentation of cell nuclei in virtual microscopy images: a minimum-model approach.," Sci. Rep., vol. 2, p. 503, Jan. 2012.

[30] H. Fatakdawala, J. Xu, A. Basavanhally, G. Bhanot, S. Ganesan, M Feldman, J. E. Tomaszewski, and A. Madabhushi, "Expectation-maximization-driven geodesic active contour with overlap resolution (EMaGACOR): application to lymphocyte segmentation on breast cancer histopathology.," IEEE Trans. Biomed. Eng., vol. 57, no. 7, pp. 1676-89, Jul. 2010.

[31] C. Jung, C. Kim, S. W. Chae, and S. Oh, "Unsupervised segmentation of overlapped nuclei using Bayesian classification.," IEEE Trans. Biomed. Eng., vol. 57, no. 12, pp. 2825-32, Dec. 2010.

[32] J. P. Vink, M. B. Van Leeuwen, C. H. M. Van Deurzen, and G. De Haan, "Efficient nucleus detector in histopathology images.," $J$. Microsc., vol. 249, no. 2, pp. 124-35, Feb. 2013.

[33] A. Friebel, J. Neitsch, T. Johann, S. Hammad, J. G. Hengstler, D. Drasdo, and S. Hoehme, "TiQuant: Software for tissue analysis, quantification and surface reconstruction," Bioinformatics, vol. 31, no. 19 , pp. 3234-3236, 2015.

[34] M. Puderbach, C. Hintze, S. Ley, M. Eichinger, H.-U. Kauczor, and J. Biederer, "MR imaging of the chest: a practical approach at 1.5T.," Eur. J. Radiol., vol. 64, no. 3, pp. 345-55, Dec. 2007.

[35] C. Hintze, A. Stemmer, M. Bock, T. A. Kuder, F. Risse, J. Dinkel, H. Prüm, M. Puderbach, C. Fink, J. Biederer, and H.-U. Kauczor, "A hybrid breath hold and continued respiration-triggered technique for time-resolved 3D MRI perfusion studies in lung cancer.," Rofo, vol. 182, no. 1, pp. 45-52, Jan. 2010.

[36] "MITK Diffusion." [Online]. Available: http://mitk.org/wiki/DiffusionImaging. [Accessed: 25-Jan-2016].

[37] M. G. Rojo, G. B. García, C. P. Mateos, J. G. García, and M. C. Vicente, "Critical comparison of 31 commercially available digital slide systems in pathology.," Int. J. Surg. Pathol., vol. 14, no. 4, pp. 285-305, Oct. 2006.

[38] J. Lotz, J. Berger, B. Müller, K. Breuhahn, N. Grabe, S. Heldmann, A. Homeyer, B. Lahrmann, H. Laue, J. Olesch, M. Schwier, O. Sedlaczek, and A. Warth, "Zooming in: high resolution 3D reconstruction of differently stained histological whole slide images," in Proceedings of the SPIE 9041, Medical Imaging 2014. Digital Pathology, 2014.

[39] M. Macenko, M. Niethammer, J. S. Marron, D. Borland, J. T. Woosley, X. Guan, C. Schmitt, and N. E. Thomas, "A method for normalizing histology slides for quantitative analysis," in Proceedings of the 2009 IEEE International Symposium on Biomedical Imaging: From Nano to Macro, ISBI 2009, 2009, pp. 1107-1110.

[40] A. C. Ruifrok and D. A. Johnston, "Quantification of histochemical staining by color deconvolution.," Anal. Quant. Cytol. Histol., vol. 23, no. 4, pp. 291-9, Aug. 2001.
[41] P. Bamford and B. Lovell, "Unsupervised cell nucleus segmentation with active contours," Signal Processing, vol. 71, no. 2, pp. 203-213, 1998.

[42] L. Yang, O. Tuzel, P. Meer, and D. J. Foran, "Automatic image analysis of histopathology specimens using concave vertex graph.," Med. Image Comput. Comput. Assist. Interv., vol. 11, no. Pt 1, pp. 833-41, Jan. 2008.

[43] E. Gladilin, S. Goetze, J. Mateos-Langerak, R. van Driel, K. Rohr, and R. Eils, "Shape normalisation of 3D cell nuclei using elastic spherical mapping," J Microsc., vol. 231, no. 1, pp. 105-114, 2008.

[44] I. Martin, B. Dozin, R. Quarto, R. Cancedda, and F. Beltrame, "Computer-based technique for cell aggregation analysis and cell aggregation in in vitro chondrogenesis," Cytometry, vol. 28, no. 2, pp. 141-146, 1997.

[45] J. H. McDonald, "Kruskal-Wallis test," in Handbook of Biological Statistics, 3rd ed., Baltimore, Maryland: Sparky House Publishing, 2014, pp. 158-165.

[46] C. Stecco, W. Hammer, A. Vleeming, and R. De Caro, Functional Atlas of the Human Fascial System. Elsevier, 2015.

[47] A. M. Khan, N. Rajpoot, D. Treanor, and D. Magee, "A nonlinear mapping approach to stain normalization in digital histopathology images using image-specific color deconvolution," IEEE Trans. Biomed. Eng., vol. 61, no. 6, pp. 1729-1738, 2014.

[48] U. Del Monte, "Does the cell number 109 still really fit one gram of tumor tissue?," Cell Cycle, vol. 8, no. 3, pp. 505-506, 2009

[49] H. Kobayashi, R. Watanabe, and P. L. Choyke, "Improving conventional enhanced permeability and retention (EPR) effects; What is the appropriate target?," Theranostics, vol. 4, no. 1, pp. 81$89,2014$.

[50] H. Boonstra, J. W. Oosterhuis, A. M. Oosterhuis, and G. J. Fleuren, "Cervical tissue shrinkage by formaldehyde fixation, paraffin wax embedding, section cutting and mounting," Virchows Arch. A Pathol. Anat. Histopathol., vol. 402, no. 2, pp. 195-201, 1983.

[51] T. Tran, C. P. Sundaram, C. D. Bahler, J. N. Eble, D. J. Grignon, M. F. Monn, N. B. Simper, and L. Cheng, "Correcting the Shrinkage Effects of Formalin Fixation and Tissue Processing for Renal Tumors: toward Standardization of Pathological Reporting of Tumor Size.," J. Cancer, vol. 6, no. 8, pp. 759-66, Jan. 2015.

[52] M. Y. K. Bilgili, "Reproductibility of apparent diffusion coefficients measurements in diffusion-weighted MRI of the abdomen with different b values," Eur. J. Radiol., vol. 81, no. 9, pp. 2066-2068, 2012.

[53] M. Jang, S. M. Kim, B. La Yun, H. S. Ahn, S. Y. Kim, E. Kang, and S. Kim, "Reproducibility of Apparent Diffusion Coefficient Measurements in Malignant Breast Masses," J. Korean Med. Sci., vol. 30 , no. 11, pp. 1689-1697, 2015.

[54] J. G. Whisenant, G. D. Ayers, M. E. Loveless, S. L. Barnes, D. C. Colvin, and T. E. Yankeelov, "Assessing reproducibility of diffusion-weighted magnetic resonance imaging studies in a murine model of HER2+ breast cancer," Magn. Reson. Imaging, vol. 32, no. 3, pp. 245-249, 2014.

[55] L. Chen, M. Liu, J. Bao, Y. Xia, J. Zhang, L. Zhang, X. Huang, and J. Wang, "The correlation between apparent diffusion coefficient and tumor cellularity in patients: A meta-analysis," PLoS One, vol. 8, no. $11,2013$.

[56] S. Maetani, "TNM staging system.," Dis. Colon Rectum, vol. 35, no. 1, p. 104, 1992 